\title{
Efficiency Resource Allocation for Device-to-Device Underlay Communication Systems: A Reverse Iterative Combinatorial Auction Based Approach
}

\author{
Chen Xu*, Lingyang Song*, Zhu Han ${ }^{\dagger}$, Qun Zhao ${ }^{\ddagger}$, Xiaoli Wang ${ }^{\ddagger}$, \\ Xiang Cheng*, and Bingli Jiao* \\ *Peking University, Beijing, China. \\ $\dagger$ University of Houston, Houston, USA. \\ $\ddagger$ DoCoMo Beijing Communications Laboratories, China.
}

\begin{abstract}
Peer-to-peer communication has been recently considered as a popular issue for local area services. An innovative resource allocation scheme is proposed to improve the performance of mobile peer-to-peer, i.e., device-to-device (D2D), communications as an underlay in the downlink (DL) cellular networks. To optimize the system sum rate over the resource sharing of both D2D and cellular modes, we introduce a reverse iterative combinatorial auction as the allocation mechanism. In the auction, all the spectrum resources are considered as a set of resource units, which as bidders compete to obtain business while the packages of the D2D pairs are auctioned off as goods in each auction round. We first formulate the valuation of each resource unit, as a basis of the proposed auction. And then a detailed non-monotonic descending price auction algorithm is explained depending on the utility function that accounts for the channel gain from D2D and the costs for the system. Further, we prove that the proposed auction-based scheme is cheat-proof, and converges in a finite number of iteration rounds. We explain non-monotonicity in the price update process and show lower complexity compared to a traditional combinatorial allocation. The simulation results demonstrate that the algorithm efficiently leads to a good performance on the system sum rate.
\end{abstract}

This paper is partially supported by US NSF CNS-1117560, ECCS-1028782, CNS-0953377, CNS-0905556, and Qatar National Research Fund. 


\section{INTRODUCTION}

As one of next-generation wireless communication systems, Third Generation Partnership Project (3GPP) Long Term Evolution (LTE) is committed to provide technologies for high data rates and system capacity. Further, LTE-Advanced (LTE-A) was defined to support new components for LTE to meet higher communication demands [1]. Local area services are considered as popular issues to be improved, and by reusing spectrum resources local data rates have been increased dramatically. However, the unlicensed spectrum reuse may bring inconvenience for local service providers to guarantee a stable controlled environment, e.g., ad hoc network [2], which is not in the control of the base station (BS) or other central nodes. Hence, accessing to the licensed spectrum has attracted much attention.

Device-to-Device (D2D) communication is a technology component for LTE-A. The existing researches allow D2D as an underlay to the cellular network to increase the spectral efficiency [1], [3]. In D2D communication, user equipments (UEs) transmit data signals to each other over a direct link using the cellular resources instead of through the BS, which differs from femtocell [4] where users communicate with the help of small low-power cellular base stations. D2D users communicate directly while remaining controlled under the BS. Therefore, the potential of improving spectral utilization has promoted much work in recent years [5]-[10], which shows that D2D can improve system performances by reusing cellular resources. As a result, D2D is expected to be a key feature supported by next generation cellular networks.

Although D2D communication brings improvement in spectral efficiency and makes large benefits on system capacity, it also causes interference to the cellular network as a result of spectrum sharing. Thus, an efficient interference coordination must be formulated to guarantee a target performance level of the cellular communication. There exists several work about the power control of D2D UEs for restricting co-channel interference [1], [3], [11], [12]. The authors in [13] utilized MIMO transmission schemes to avoid interference from cellular downlink to D2D receivers sharing the same resources, which aims at guaranteeing D2D performances. Interference management both from cellular to D2D communication and from D2D to cellular networks are considered in [14]. In order to further improve the gain from 
intra-cell spectrum reuse, properly pairing the cellular and D2D users for sharing the same resources has been studied [15], [16]. The authors in [16] proposed an alternative greedy heuristic algorithm to lessen interference to the primary cellular networks using channel state information (CSI). The scheme is easy-operated but cannot prevent signaling overhead. In [17], the resource allocation scheme avoids the harmful interference by tracking the near-far interference, identifies the interfering cellular users, and makes the uplink (UL) frequency bands efficiently used. Also, the target is to prevent interference from cellular to D2D communication. In [18], the authors provided analysis on optimum resource allocation and power control between the cellular and D2D connections that share the same resources for different resource sharing modes, and evaluated the performance of the D2D underlay system in both a single cell scenario and the Manhattan grid environment. Then, the schemes are to further optimize the resource usage among users sharing the same resources. Based on the aforementioned work, it indicates that by proper resource management, D2D communication can effectively improve the system throughput with the interference between cellular networks and D2D transmissions being restricted. However, the problem of allocating cellular resources to D2D transmissions is of great complexity. Our works differ from all mentioned above in that we consider a scheme to maximize the system sum rate by allowing multiple pairs share one cellular user's spectrum resource.

Game theory offers a set of mathematical tools to study the complex interactions among interdependent rational players and to predict their choices of strategies [19]. In the present researches, game theory including a large number of different game methods are used to analyze resource allocation problems, such as power and wireless spectrum allocations in communication networks [20], resource management in grids [21], and distributed resource coordination in mega-scale container terminal [22]. In [20], the authors proposed a sequential auction for sharing the wireless resource, which is managed by a spectrum broker that collects bids and allocates discrete resource units using a sequential second-price auction. A combinatorial auction model for resource management was introduced in [21], [22]. The combinatorial auction-based resource allocation mechanism allows an agent (bidder) to place bids on combinations of resources, called "packages", rather than just individual resource 
unit.

Actually, the combinatorial auctions (CAs) have been employed in a variety of industries for, e.g., truckload transportation, airport arrival and departure slots, as well as wireless communication services. The benchmark environment of auction theory is the private value model, introduced by Vickrey (1961), in which one bidder has a value for each package of items and the value is not related to the private information of other bidders [23]. Much of work has not recognized that bidders care in complex ways about the items they compete. The CAs motivate bidders to fully express their preferences, which is an advantage in improving system efficiency and auction revenues. Up to that point, our interest is to apply the CA game in solving arbitrary D2D links reusing the same cellular frequency bands with the purpose of optimizing the system capacity.

However, it exists a series of problems and challenges in CAs, such as pricing and bidding rules, the winner determination problem (WDP) which, as mentioned in the literature, leads to the NP-hard allocation problem. Therefore, we focus on the evolution mechanisms named iterative combinatorial auctions (I-CAs) [24], [25]. In I-CAs, the bidders submit multiple bids iteratively, and the auctioneer computes provisional allocations and ask prices in each auction round.

In this paper, we study an effective spectrum resource allocation for D2D communication as an underlay to further improve system efficiency based on the I-CA. The whole system consists of the BS, multiple cellular users that receive signals from the BS, and multiple D2D pairs that communicate with respective receivers using licensed spectrum resources. Considering that interference minimization is a key point and multiple D2D pairs sharing the same resources can bring large benefits on system capacity, we formulate the problem as a reverse I-CA game. That means, the resources as the bidders compete to obtain business, while D2D links as the goods or services wait to be sold. By this way, the packages of D2D pairs are auctioned off in each auction round. Furthermore, we investigate some important properties of the proposed resource allocation mechanism such as cheat-proof, convergence and pricemonotonicity. Part of our work has been published in [26], which introduces a sequential second price auction as the allocation mechanism for D2D underlay communication, and 
explains the detailed algorithm using an $\mathrm{N}$-ary tree. In this work, we further reduce the computational complexity and apply our scheme to WINNER II channel models [27] which contain a well-known indoor scenario. The simulation results show that the auction algorithm leads to a good performance on the system sum rate, and provides high system efficiency while has lower complexity than the exhaustive search allocation.

The rest of the paper is organized as follows: In Section II, we describe the system model of the D2D communication underlaying cellular network, and give the explanation and expression of the system sum rate. The primary problem is formulated in Section III] In Section IV, the resource allocation algorithm based on a reverse I-CA is proposed. In Section V, the main properties of the proposed algorithm are investigated. In Section VI, we present the numerical simulation results and relevant analysis on the system sum rate, algorithm efficiency, and properties. Finally, we draw the conclusions in Section VII.

\section{System ModeL}

In this section, we introduce the system model for D2D underlay communication. The scenario of multiple D2D and cellular users is first described, and then, the expression of system sum rate is given.

\section{A. Scenario Description}

A model of a single cell with multiple users is considered. As shown in Fig. 1, UEs with data signals between each other are in the D2D communication mode while UEs that transmit data signals with the BS keep in the traditional cellular mode. Each user is equipped with a single omnidirectional antenna. The locations of cellular users and D2D pairs are randomly set and traversing the whole cell. Without loss of generality, we employ the uniform distribution to describe the user locations which is proposed for system simulation in [28]. Notice that from stochastic geometry with for Poisson distributions, the users are uniformly located as well if the number of users is known [29]. For simplicity and clarity, we illustrate co-channel interference scenario involving three $\mathrm{UEs}\left(\mathrm{UE}_{c}, \mathrm{UE}_{d, 1}\right.$ and $\left.\mathrm{UE}_{d, 2}\right)$, and omit the interference and control signal signs among others. $\mathrm{UE}_{c}$ is a traditional cellular user that is distributed uniformly in the cell. $\mathrm{UE}_{d, 1}$ and $\mathrm{UE}_{d, 2}$ are close enough to satisfy the distance constraints 
of $\mathrm{D} 2 \mathrm{D}$ communication, and at the same time they also have communicating demands. One member of the D2D pair $\mathrm{UE}_{d, 1}$ is distributed uniformly in the cell, and the position of the other member $\mathrm{UE}_{d, 2}$ follows a uniform distribution inside a region at most $L$ from $\mathrm{UE}_{d, 1}$.

The existing researches [17], [18] confirm that with power control or resource scheduling mechanism, the inter-cell interference can be managed efficiently. Therefore, we place an emphasis on the intra-cell interference, which is due to resource sharing of D2D and cellular communication. Generally speaking, the session setup of D2D communication requires the following steps [1]:

1) A request of communicating is initiated by one UE pair.

2) The system detects traffic originating from and destined to the UE in the same subnet.

3) If the traffic fulfills a certain criterion (e.g., data rate), the system considers the traffic as the potential D2D traffic.

4) The BS checks if D2D communication offers higher throughput.

5) If both UEs are D2D capable and D2D communication offers higher throughput, the BS may set up a D2D bearer.

The cross-layer processes of resource control can be contained in the above steps, and be generally summarized as: the transmitters (both cellular and D2D users) send detecting signals. Then CSI would be obtained by corresponding receivers and be feedback to the control center (e.g. the BS). The power control and spectrum allocation are conducted based on certain principles. Finally, the BS sends control signals to users according to allocation results.

Even if the D2D connection setup is successful, the BS still maintain detecting if UE should be back to the cellular communication mode. Furthermore, the BS maintains the radio resource control for both cellular and D2D communication. Based on these communication features, our work mainly focuses on assigning cellular resources to D2D communication.

In this paper, we consider a scenario of sharing downlink (DL) resource of the cellular network as shown in Fig. 1. We assume $\mathrm{UE}_{d, 1}$ is the transmitter of the D2D pair sharing the same sub-channel with the $\mathrm{BS}$, and thus, $\mathrm{UE}_{d, 2}$ as the $\mathrm{D} 2 \mathrm{D}$ receiver receives interference from the $\mathrm{BS}$. Also, the cellular receiver $\mathrm{UE}_{c}$ is exposed to interference from $\mathrm{UE}_{d, 1}$. In addition, 
the D2D users feed back the CSI to the BS, whereas the BS transmits control signals to the D2D pair, in the way that the system achieves D2D power control and resource allocation.

During the DL period of the cellular system, both cellular and D2D users receive interference as they share the same sub-channels. Here, we assume that any cellular user's resource blocks (RBs) can be shared with multiple D2D pairs and each pair can use more than one user's RBs for transmitting. We assume the numbers of cellular users and D2D pairs in the model are $C$ and $D$, respectively. During the DL period, the BS transmits signal $x_{c}$ to the $c$-th $(c=1,2, \ldots, C)$ cellular user, and the $d$-th $(d=1,2, \ldots, D) \mathrm{D} 2 \mathrm{D}$ pair uses the same spectrum resources transmitting signal $x_{d}$. The received signals at UE $c$ and D2D receiver $d$ are written as

$$
\begin{gathered}
y_{c}=\sqrt{P_{B}} h_{B c} x_{c}+\sum_{d} \beta_{c d} \sqrt{P_{d}} h_{d c} x_{d}+n_{c}, \\
y_{d}=\sqrt{P_{d}} h_{d d} x_{d}+\sqrt{P_{B}} h_{B d} x_{c}+\sum_{d^{\prime}} \beta_{d d^{\prime}} \sqrt{P_{d^{\prime}}} h_{d^{\prime} d} x_{d^{\prime}}+n_{d},
\end{gathered}
$$

where $P_{B}, P_{d}$ and $P_{d^{\prime}}$ are the transmit power of BS, D2D transmitter $d, d^{\prime}$, respectively. $h_{i j}$ is the channel response of the $i-j$ link that is from equipments $i$ to $j . n_{c}$ and $n_{d}$ are the additive white Gaussian noise (AWGN) at the receivers with one-sided power spectral density (PSD) $N_{0} \cdot \beta_{c d}$ represents the presence of interference satisfying $\beta_{c d}=1$ when RBs of UE $c$ are assigned to UE $d$, otherwise $\beta_{c d}=0$. As a cellular user can share resources with multiple D2D pairs, it also satisfies $0 \leq \sum_{d} \beta_{c d} \leq D$. Similarly, $\beta_{d d^{\prime}}$ represents the presence of interference between D2D pairs $d$ and $d^{\prime}$.

In this paper, the channel is modeled as the Rayleigh fading channel, and thus, the channel response follows the independent itentical complex Gaussian distribution. In addition, the free space propagation path-loss model, $P=P_{0} \cdot\left(d / d_{0}\right)^{-\alpha}$, is used where $P_{0}$ and $P$ represent signal power measured at $d_{0}$ and $d$ away from the transmitter, respectively. $\alpha$ is the path-loss exponent. Hence, the received power of each link can be expressed as

$$
P_{r, i j}=P_{i} \cdot h_{i j}^{2}=P_{i} \cdot\left(d_{i j}\right)^{-\alpha} \cdot h_{0}^{2},
$$

where $P_{r, i j}$ and $d_{i j}$ are the received power and the distance of the $i-j$ link, respectively. $P_{i}$ represents the transmit power of equipment $i$, and $h_{0}$ is the complex Gaussian channel 
coefficient that obeys the distribution $\mathcal{C N}(0,1)$. Besides, we simplify the received power at $d_{0}=1$ equals the transmit power.

\section{B. System Sum Rate}

For the purpose of maximizing the network capacity, the signal to interference plus noise ratio (SINR) should be considered as an important indicator. The SINR of user $j$ is

$$
\gamma_{j}=\frac{P_{i} h_{i j}^{2}}{P_{\text {int }, j}+N_{0}}
$$

where $P_{\mathrm{int}, j}$ denotes the interference signal power received by user $j$, and $N_{0}$ accounts for the terminal noise at the receiver.

Determined by the Shannon capacity formula, we can calculate the channel rate corresponding to the SINR of cellular and D2D users. As cellular users suffer interference from D2D communicating that sharing the same spectrum resource, the interference power of cellular user $c$ is

$$
P_{\text {int }, c}=\sum_{d} \beta_{c d} P_{d} h_{d c}^{2}
$$

While the interference of $\mathrm{D} 2 \mathrm{D}$ receiver $d$ is from both $\mathrm{BS}$ and $\mathrm{D} 2 \mathrm{D}$ users that are assigned the same resources to, the interference power of user $d$ can be expressed as

$$
P_{\text {int }, d}=P_{B} h_{B d}^{2}+\sum_{d^{\prime}} \beta_{d d^{\prime}} P_{d^{\prime}} h_{d^{\prime} d}^{2} .
$$

Based on (4), (5), and (6), we can obtain the channel rate of cellular user $c$ and D2D receiver $d$ as

$$
\begin{gathered}
R_{c}=\log _{2}\left(1+\frac{P_{B} h_{B c}^{2}}{\sum_{d} \beta_{c d} P_{d} h_{d c}^{2}+N_{0}}\right), \\
R_{d}=\log _{2}\left(1+\frac{P_{d} h_{d d}^{2}}{P_{B} h_{B d}^{2}+\sum_{d^{\prime}} \beta_{d d^{\prime}} P_{d^{\prime}} h_{d^{\prime} d}^{2}+N_{0}}\right),
\end{gathered}
$$

respectively. Here, $d \neq d^{\prime}$. So $\sum_{d^{\prime}} \beta_{d d^{\prime}} P_{d^{\prime}} h_{d^{\prime} d}^{2}$ represents the interference from the other D2D pairs that share spectrum resources with pair $d$.

The DL system sum rate can be defined as

$$
\Re=\sum_{c=1}^{C}\left(R_{c}+\sum_{d=1}^{D} \beta_{c d} R_{d}\right) .
$$


In the next section, we formulate the problem of designing $\beta_{c d}$ for each D2D pair as an optimization issue of maximizing $\Re$.

\section{PROBLEM Formulation}

In this section, we introduce two concepts: valuation model and utility function, which are bases of the auction mechanism. Also, some definitions are given.

\section{A. Valuation Model}

As D2D communication shares the same spectrum resources with cellular communication at the same time slot, the co-channel interference should be limited as much as possible to optimize the system performance. The radio signals experience different degrees of fading, and thus, the amount of interference depends on transmit power and spatial distances. Accordingly, we focus on assigning appropriate resource blocks (RBs) occupied by cellular users to D2D pairs in order to minimize interference to achieve a higher system sum rate. Next, we formulate the relation between the allocation result and the rate of the shared channel. The relation can be defined as a value function whose target value is the channel rate.

We define $\mathcal{D}$ as a package of variables representing the index of D2D pairs that share the same resources. We assume the total pairs can form $N$ such packages. Thus, if the members of the $k$-th $(k=1,2, \ldots, N)$ D2D user package share resources with cellular user $c$, the channel rates of UE $c$ and D2D pair $d\left(d \in \mathcal{D}_{k}\right)$ can be written as

$$
\begin{gathered}
R_{c}^{k}=\log _{2}\left(1+\frac{P_{B} h_{B c}^{2}}{\sum_{d \in \mathcal{D}_{k}} P_{d} h_{d c}^{2}+N_{0}}\right), \\
R_{d}^{k}=\log _{2}\left(1+\frac{P_{d} h_{d d}^{2}}{P_{B} h_{B d}^{2}+\sum_{d^{\prime} \in \mathcal{D}_{k}-\{d\}} P_{d^{\prime}} h_{d^{\prime} d}^{2}+N_{0}}\right),
\end{gathered}
$$

respectively. The rate of the operating channel shared by UE $c$ and D2D pairs $d \in \mathcal{D}_{k}$ is

$$
R_{c k}=R_{c}^{k}+\sum_{d \in \mathcal{D}_{k}} R_{d}^{k}
$$


According to (10) $~(12)$, when assigning resources of UE $c$ to the $k$-th package of D2D pairs, the channel rate is given by

$$
V_{c}(k)=\log _{2}\left(1+\frac{P_{B} h_{B c}^{2}}{\sum_{d \in \mathcal{D}_{k}} P_{d} h_{d c}^{2}+N_{0}}\right)+\sum_{d \in \mathcal{D}_{k}} \log _{2}\left(1+\frac{P_{d} h_{d d}^{2}}{P_{B} h_{B d}^{2}+\sum_{d^{\prime} \in \mathcal{D}_{k}-\{d\}} P_{d^{\prime}} h_{d^{\prime} d}^{2}+N_{0}}\right) .
$$

In the proposed reverse I-CA mechanism, we consider spectrum resources occupied by cellular user $c$ as one of the bidders who submit bids to compete for the packages of D2D pairs, in order to maximize the channel rate. It is obvious that there would be a gain of channel rate owing to D2D communicating as long as the contribution to data signals from D2D is larger than that to interference signals. Considering the constraint of a positive value, we define the performance gain as

$$
v_{c}(k)=\max \left(V_{c}(k)-V_{c}, 0\right),
$$

which is the private valuation of bidder $c$ for the package of D2D pairs $\mathcal{D}_{k}$. Here, $V_{c}$ denotes the channel rate of UE $c$ without co-channel interference and is obtained by

$$
V_{c}=\log _{2}\left(1+\frac{P_{B} h_{B c}^{2}}{N_{0}}\right) .
$$

Thus, we have the following definition:

Definition 1: A valuation model $\mathcal{V}=\left\{v_{c}(k)\right\}$ is a set of the private valuations of all bidders $c \in\{1,2, \ldots, C\}$ for all packages $\mathcal{D}_{k} \subseteq\{1,2, \ldots, D\}(k \in\{1,2, \ldots, N\})$.

\section{B. Utility Function}

In the auction, the cellular resource denoted by $c$ obtains a gain by getting a package of D2D communications. However, there exists some cost such as control signals transmission and information feedback during the access process. We define the cost as a pay price.

Definition 2: The price to be payed by the bidder $c$ for the package $\mathcal{D}_{k}$ is called pay price denoted by $\mathcal{P}_{c}(k)$. The unit price of item $d\left(\forall k, d \in \mathcal{D}_{k}\right)$ can be denoted by $p_{c}(d)$.

Here, we consider linear anonymous prices [24], which means the prices are linear if the price of a package is equal to the sum of the prices of its items, and the prices are anonymous 
if the prices of the same package for different bidders are equal. Thus, we have

$$
\mathcal{P}_{c}(k)=\sum_{d \in \mathcal{D}_{k}} p_{c}(d)=\sum_{d \in \mathcal{D}_{k}} p(d), \forall c=1,2, \ldots, C .
$$

Therefore, the payment of a bidder is determined by the unit price $p(d)$ and the size of bidding package $\mathcal{D}_{k}$.

Definition 3: Bidder utility, or named bidder payoff $\mathcal{U}_{c}(k)$ expresses satisfaction of bidder $c$ getting package $\mathcal{D}_{k}$. The bidder utility can be defined as

$$
\mathcal{U}_{c}(k)=v_{c}(k)-\mathcal{P}_{c}(k) .
$$

Based on (14), (16), (17), $V_{c}(k)$ in (13) and $V_{c}$ in (15), we can obtain the utility of bidder $c$ as

$$
\begin{aligned}
\mathcal{U}_{c}(k)= & \log _{2}\left(1+\frac{P_{B} h_{B c}^{2}}{\sum_{d \in \mathcal{D}_{k}} P_{d} h_{d c}^{2}+N_{0}}\right)+\sum_{d \in \mathcal{D}_{k}} \log _{2}\left(1+\frac{P_{d} h_{d d}^{2}}{P_{B} h_{B d}^{2}+\sum_{d^{\prime} \in \mathcal{D}_{k}-\{d\}} P_{d^{\prime}} h_{d^{\prime} d}^{2}+N_{0}}\right) \\
& -\log _{2}\left(1+\frac{P_{B} h_{B c}^{2}}{N_{0}}\right)-\sum_{d \in \mathcal{D}_{k}} p(d)
\end{aligned}
$$

In order to describe the allocation outcome intuitively, we give the definition below.

Definition 4: The result of the auction is a spectrum allocation denoted by $\mathcal{X}=\left(X_{1}, X_{2}, \ldots, X_{C}\right)$, which allocates a corresponding package to each bidder. And the allocated packages may not intersect $\left(\forall i, j, X_{i} \cap X_{j}=\emptyset\right)$.

We consider a set of binary variables $\left\{x_{c}(k)\right\}$ to redefine the allocation as

$$
x_{c}(k)=\left\{\begin{array}{lc}
1, & \text { if } X_{c}=\mathcal{D}_{k}, \\
0, & \text { otherwise. }
\end{array}\right.
$$

According to the literature, two most popular bidding languages are exclusive-OR (XOR), which allows a bidder to submit multiple bids but at most one of the bids can win, and additive-OR (OR), which allows one to submit multiple bids and any non-intersecting combination of the bids can win. We consider the XOR bidding language in this paper. Thus, (19) satisfies $\sum_{k=1}^{N} x_{c}(k) \leq 1$ and $\sum_{k=1}^{N} x_{c}(k)=0 \Rightarrow X_{c}=\emptyset$ for $\forall c=1,2, \ldots, C$. If given an allocation $\mathcal{X}$, the total bidder utility of all bidders can be denoted as $\mathcal{U}_{\text {all }}(\mathcal{X})=$ $\sum_{c=1}^{C} \sum_{k=1}^{N} x_{c}(k) \mathcal{U}_{c}(k)$. Furthermore, the auctioneer revenue is denoted as $\mathcal{A}(\mathcal{X})=$ $\sum_{c=1}^{C} \sum_{k=1}^{N} x_{c}(k) \mathcal{P}_{c}(k)$, which is usually considered to be the auctioneer's gain. 


\section{Resource Allocation Algorithm Based on Reverse Iterative}

\section{COMBINATORIAL AUCTION}

In this section, we formulate the resource allocation for D2D communication as a reverse I-CA game. First, we introduce some concepts of the I-CA games. Then, we investigate details of the allocation process.

\section{A. Reverse Iterative Combinatorial Auction Game}

As mentioned before, we assume the total spectrum resources are divided into $C$ units with each one already providing communication service to one cellular user. By the auction game, the spectrum units are assigned to $N$ user packages $\left\{\mathcal{D}_{1}, \mathcal{D}_{2}, \ldots, \mathcal{D}_{N}\right\}$, with each package consisting of at least one D2D pair. In other words, the spectrum units compete to obtain D2D communication for improving the channel rate.

During an I-CA game, the auctioneer announces an initial price for each item, and then, the bidders submit to the auctioneer their bids at the current price. As long as the demand exceeds the supply, or on the contrary that the supply exceeds the demand, the auctioneer updates (raises or reduces) the corresponding price and the auction goes to the next round.

Obviously, it can be shown that the overall gain, which includes the total gain of the auctioneer and all bidders does not depend on the pay price, but equals to the sum of the allocated packages' valuations, i.e.,

$$
\begin{aligned}
\mathcal{A}(\mathcal{X})+\mathcal{U}_{\text {all }}(\mathcal{X}) & =\sum_{c=1}^{C} \sum_{k=1}^{N} x_{c}(k) \mathcal{P}_{c}(k)+\sum_{c=1}^{C} \sum_{k=1}^{N} x_{c}(k) \mathcal{U}_{c}(k) \\
& =\sum_{c=1}^{C} \sum_{k=1}^{N} x_{c}(k) \mathcal{P}_{c}(k)+\sum_{c=1}^{C} \sum_{k=1}^{N} x_{c}(k)\left[\left(v_{c}(k)-\mathcal{P}_{c}(k)\right)\right] \\
& =\sum_{c=1}^{C} \sum_{k=1}^{N} x_{c}(k) v_{c}(k) .
\end{aligned}
$$

As our original intention, we employ the I-CA to obtain an efficient allocation for spectrum resources.

Definition 5: An efficient allocation denoted by $\tilde{\mathcal{X}}=\left(\tilde{X}_{1}, \tilde{X}_{2}, \ldots, \tilde{X}_{C}\right)=\left\{\tilde{x}_{c}(k)\right\}$ is an allocation that maximizes the overall gain. 
Given the private bidder valuations for all possible packages in (14), an efficient allocation can be obtained by solving the combinatorial allocation problem (CAP).

Definition 6: The Combinatorial Allocation Problem (CAP), also sometimes referred as Winner Determination Problem (WDP), leads to an efficient allocation by maximizing the overall gain: $\max _{\mathcal{D}_{k}=X_{c} \in \mathcal{X} \in \mathscr{X}} \sum_{c=1}^{C} v_{c}(k)$, where $\mathscr{X}$ denotes the set of all possible allocations.

An integer linear program using the binary decision variables $\left\{x_{c}(k)\right\}$ is formulated for the CAP as

$$
\begin{aligned}
& \max \sum_{c=1}^{C} \sum_{k=1}^{N} x_{c}(k) v_{c}(k), \\
\text { s.t. } & \sum_{k=1}^{N} x_{c}(k) \leq 1, \forall c \in\{1,2, \ldots, C\}, \\
& \sum_{\mathcal{D}_{k}: d \in \mathcal{D}_{k}} \sum_{c=1}^{C} x_{c}(k) \leq 1, \forall d \in\{1,2, \ldots, D\} .
\end{aligned}
$$

The objective function maximizes the overall gain, and the constraints guarantee: 1) at most one package can be allocated to each bidder; 2) each item cannot be sold more than once.

In fact, there might be multiple optimal solutions of the CAP with the same objective function. From the auctioneer's point of view, tie-breaking rules are needed to determine which of the optimal solutions is selected. In a real auction, the auctioneer does not know the private valuations of the bidders, neither can it solve the NP-hard problem. To solve the CAP, the auctioneer selects the winners on the basis of the submitted bids in each round. Therefore, in case of the XOR bidding language, the WDP formulation is similar to the CAP and the only difference is the objective function

$$
\max \sum_{c=1}^{C} \sum_{k=1}^{N} x_{c}(k) \mathcal{P}_{c}^{t}(k),
$$

where $\mathcal{P}_{c}^{t}(k)$ represents the pay price of bidder $c$ for package $\mathcal{D}_{k}$ in round $t$.

Based on Definition 5, the overcome of a CA is not always efficient. Here, we employ allocating efficiency as a primary measure to benchmark auctions.

Definition 7: Allocating efficiency in CAs can be expressed as the ratio of the overall 
gain of the final allocation to that of an efficient allocation [24]

$$
\mathcal{E}(\mathcal{X})=\frac{\mathcal{A}(\mathcal{X})+\mathcal{U}_{\text {all }}(\mathcal{X})}{\mathcal{A}(\tilde{\mathcal{X}})+\mathcal{U}_{\text {all }}(\tilde{\mathcal{X}})}
$$

which has $\mathcal{E}(\mathcal{X}) \in[0,1]$.

\section{B. Algorithm for Resource Allocation}

In this subsection, the details of the allocation scheme based on reverse I-CA are introduced. We has modeled the D2D resource allocation problem as a reverse I-CA game and gave the valuation model, utility function and other important concepts. Many I-CA designs, especially for the centralized I-CA design, are based on ask prices. The price-based I-CA designs differ by the pricing scheme and price update rules. In the proposed algorithm, linear prices are used as mentioned in Subsection $\amalg$ II-B for they are easy to understand for bidders and convenient to communicate in each auction round. Because of the interference from D2D links, cellular channels should guarantee the performance of cellular system before allowing the D2D access. Hence, we consider a descending price criterion in the algorithm. Prices update by a greedy mode that once a bidder submits a bid for items or packages the corresponding prices are fixed, otherwise the prices are decreased.

At the beginning of the allocation, the BS collects the location information of all the D2D pairs. In addition, the round index $t=0$, the initial ask price $p^{0}(d)$ for each item (D2D pair) $d$, and the fixed price reduction $\Delta>0$ are set up. When the initial prices are announced to all the bidders (i.e. spectrum resources occupied by cellular UEs), each bidder submits bids, which consist of its desired packages and the corresponding pay prices. Jump bidding where bidders are allowed to bid higher than the prices, is not allowed in our scheme, thus bidders always bid at the current prices. According to the CAP proposed in Definition 6 and the analysis about the WDP, we simplify the problem of maximizing the overall gain as a process of collecting the highest pay price. As a result, bidder $c$ bids for package $\mathcal{D}_{k}$ as long as $\mathcal{U}_{c}(k) \geq 0$. Combining (16) and (17), we have

$$
v_{c}(k) \geq \mathcal{P}_{c}^{t}(k)=\sum_{d \in \mathcal{D}_{k}} p^{t}(d)
$$


where the round index $t \geq 0$. In this case, let $b_{c}^{t}(k)=\left\{\mathcal{D}_{k}, \mathcal{P}_{c}^{t}(k)\right\}$ denote the submitted bid at the end of round $t$, and $\mathcal{B}^{t}=\left\{b_{c}^{t}(k)\right\}$ denotes all the bids. When (24) is not satisfied, bid $b_{c}^{t}(k)=\{\emptyset, 0\}$.

If $\exists d \in \mathcal{D}_{k}$ satisfies $\forall b_{c}^{t}(k) \in \mathcal{B}^{t}, \mathcal{D}_{k} \notin b_{c}^{t}(k)$, it reveals that the supply exceeds the demand. Then, the BS sets $t=t+1, p^{t+1}(d)=p^{t}(d)-\Delta$ where $d$ is the over-supplied item, and the auction moves on to the next round.

In a normal case, as long as the price of a package decreases below a bidder's valuation for that package, the bidder submits a bid for it. The BS allocates the package to the bidder, and fixes the corresponding prices of items. At the same time, constrained by the XOR bidding language, the bidder is not allowed to participate the following auction rounds. As the asking prices decrease discretely every round, it may exist a situation that more than one bidders bid for packages containing the same items simultaneously. The BS detects the bids of all the bidders: 1) it exists $b_{c_{1}}^{t}(k)=b_{c_{2}}^{t}(k) \neq\{\emptyset, 0\}\left(c_{1} \neq c_{2}, k \in\{1,2, \ldots, N\}\right)$; 2) it exists $b_{c_{1}}^{t}\left(k_{1}\right)=\left\{\mathcal{D}_{k_{1}}, \mathcal{P}_{c_{1}}^{t}\left(k_{1}\right)\right\}, b_{c_{2}}^{t}\left(k_{2}\right)=\left\{\mathcal{D}_{k_{2}}, \mathcal{P}_{c_{2}}^{t}\left(k_{2}\right)\right\}\left(k_{1} \neq k_{2}, c_{1}, c_{2} \in\{1,2, \ldots, C\}\right)$ satisfying $\mathcal{D}_{k_{1}} \cap \mathcal{D}_{k_{2}} \neq \emptyset$. If either of the above conditions is satisfied, the overall demand exceeds supply for at least one item. Then, the BS sets a fine tuning $p^{t}(d)=p^{t}(d)+\delta$ where $d$ is the temporary over-demanded item, and $\delta$ can be set by $\delta=\Delta / i$ where $i$ is an integer factor that affects the convergence rate. The allocation can be determined by multiple iterations.

The auction continues until all the D2D links are auctioned off or every channel wins a package. Our algorithm is detailed in Table @.

\section{Analysis of the Proposed Resource Allocation Algorithm}

In this section, we investigate the important properties of the proposed auction-based resource allocation mechanism.

\section{A. Cheat-Proof}

As the general definition, cheat-proof means that reporting the true demand in each auction round is a best response for each bidder.

Proposition 1: The resource allocation algorithm based on the reverse I-CA is cheat-proof. 
Proof: From (18), we can get that the utility of bidder $\mathcal{U}_{c}(k)$ depends on the valuation of the package it bids and unit prices of the items. In details, it is the interference (between cellular and D2D communications) that mainly affects the utility. As the expression is extremely complex to resolve, we consider the case that only one item constitutes the package without loss of generality. The utility of bidder $c$ can be rewritten as

$\mathcal{U}_{c}(d)=\log _{2}\left(1+\frac{P_{B} h_{B c}^{2}}{P_{d} h_{d c}^{2}+N_{0}}\right)+\log _{2}\left(1+\frac{P_{d} h_{d d}^{2}}{P_{B} h_{B d}^{2}+N_{0}}\right)-\log _{2}\left(1+\frac{P_{B} h_{B c}^{2}}{N_{0}}\right)-p^{t}(d)$,

and the differential expressions of the utility with respect to $h_{d c}$ and $h_{B d}$ are

$$
\begin{gathered}
\frac{\partial \mathcal{U}_{c}(d)}{\partial h_{d c}}=\frac{-2 P_{d} h_{d c} P_{B} h_{B c}^{2}}{\ln 2\left(P_{d} h_{d c}^{2}+P_{B} h_{B c}^{2}+N_{0}\right)\left(P_{d} h_{d c}^{2}+N_{0}\right)}<0, \\
\frac{\partial \mathcal{U}_{c}(d)}{\partial h_{B d}}=\frac{-2 P_{B} h_{B d} P_{d} h_{d d}^{2}}{\ln 2\left(P_{B} h_{B d}^{2}+P_{d} h_{d d}^{2}+N_{0}\right)\left(P_{B} h_{B d}^{2}+N_{0}\right)}<0,
\end{gathered}
$$

respectively. Accordingly, utility $\mathcal{U}_{c}(d)$ is a monotonically decreasing function with respect to both $h_{d c}$ and $h_{B d}$. Thus, the optimal strategy is to bid the D2D link that has a lower channel gain with the cellular transmitter and receiver.

In a descending price auction, items are always too expensive to afford at the beginning. With the number of iterations $t$ increasing, the prices of items drop off. Given a package $\mathcal{D}_{k}$ in round $t$, bidder $c$ has the right to submit bid $\left\{\mathcal{D}_{k}, \mathcal{P}_{c}^{t}(k)\right\}$ or $\{\emptyset, 0\}$. Given that all the other bidders submit their true demands according to (24), we consider the strategy of bidder $c$ in two cases: 1) if $c$ bids $\{\emptyset, 0\}$ when its true valuation for $\mathcal{D}_{k}$ satisfies $\mathcal{U}_{c}(k) \geq 0$, it will quit this round and lose the package which maximizes its channel rate; 2) if $c$ bids $\left\{\mathcal{D}_{k}, \mathcal{P}_{c}^{t}(k)\right\}$ when its true valuation for $\mathcal{D}_{k}$ satisfies $\mathcal{U}_{c}(k)<0$ and finally wins the package, it will obviously get a negative surplus that is unwanted.

From the above analysis, we can conclude that the optimal strategy for cellular channel $c$ is to submit its true demand in each round, or it will get a loss in its utility as a result of any deceiving. That is, the proposed resource allocation algorithm is cheat-proof.

\section{B. Convergence}

In this subsection, we prove that the proposed algorithm has the convergence property. 
Proposition 2: The resource allocation algorithm based on the reverse I-CA has the convergence property that the number of the iterations is finite.

Proof: : According to Theorem 1, all the bidders submit their true demands in each auction round, in order to obtain the utility from winning. From (18), we can derive

$$
\mathcal{U}_{c}^{t+1}-\mathcal{U}_{c}^{t}=\Delta>0
$$

where $\mathcal{U}_{c}^{t}$ denotes the utility of bidder $c$ in round $t$. According to the algorithm, we have that bidder $c$ will get zero utility with no bid if $\mathcal{U}_{c}^{t}<0$, and have an opportunity to win a positive utility with bid $\left\{\mathcal{D}_{k}, \mathcal{P}_{c}^{t}(k)\right\}$ if $\mathcal{U}_{c}^{t} \geq 0$. Therefore, in the beginning, bidder $c$ plays a waiting game, and once $\mathcal{U}_{c}^{t}(k) \geq 0$, it will bid for $\mathcal{D}_{k}$. As long as it is the only one that submits a bid, it will get the package. With a sufficiently large $t$ and $\Delta>0$, we can finally get $x_{c}(k)=1$. Similarly, if more than one bidders bid for the same item, we can have an allocation by ascending price process with the step $\delta<\Delta$. Subjected to $\sum_{\mathcal{D}_{k}: d \in \mathcal{D}_{k}} \sum_{c=1}^{C} x_{c}(k) \leq 1$ in (21), the package can not be sold once more. Thus, for a finite number of packages $N$, the number of iterations is finite. That is, the proposed scheme would reach convergence.

In addition, the value of the price step $\Delta$ has a direct impact on the speed of convergence of the proposed scheme. The scheme converges fast when $\Delta$ is large, while it converges slowly when $\Delta$ is small. The fine tuning $\delta$ also has the same nature, but less impact on convergence.

\section{Price Monotonicity}

In an I-CA game, the price updates through several ways, i.e., monotonically increasing, monotonically decreasing and non-monotonic modes. Here, we focus on the price nonmonotonicity in the proposed reverse I-CA algorithm.

Proposition 3: In the proposed descending price auction, the raising item prices in a round may be necessary to reflect the competitive situation. Moreover, it brings efficiency improvement.

Proof: From the algorithm proposed in Table I, there exists a situation that more than one bidders submit bids for the same package or different packages with intersection when prices are reduced to some certain values. But auctions do not allow one item being obtained 
by multiple bidders as the second constraint in (21) shows. In this situation, raising the corresponding prices by a fine tuning $\delta=\Delta / i$ makes bidders to reinspect their utility functions. Once a bidder finds its utility less than zero, it quits from the competition. By a finite number of iterations, the winner converges to one bidder. Since the ascending price process maximizes the auctioneer revenue as shown in (22), the allocation has higher efficiency than a random allocation in that situation.

\section{Complexity}

As mentioned before, a traditional CAP in fact is an NP-hard problem, the normal solution of which is the centralized exhaustive search. We set that the number of items to be allocated is $m$, and the number of bidders is $n$. For an exhaustive optimal algorithm, an item can be allocated with $n$ possible results. Thus, all the $m$ items are allocated with $n^{m}$ possible results. The complexity of the algorithm can be denoted by $\mathcal{O}\left(n^{m}\right)$. In the proposed reverse I-CA scheme, bidders reveal their entire utility function, i.e., they calculate valuations for all possible packages, the number of which is $\mathcal{C}_{m}^{1}+\mathcal{C}_{m}^{2}+\cdots+\mathcal{C}_{m}^{m}=2^{m}-1$. If the total number of iterations is $t$, the complexity of the auction-based scheme is $\mathcal{O}\left(n\left(2^{m}-1\right)+t\right)$. From the proposed algorithm, we have $p^{t}(d)=p^{0}(d)-\Delta \cdot t \geq 0$ (The fine tuning has a small impact on the result and can be omitted here). So the worst case is $t=p^{0}(d) / \Delta$. It is obvious that for sufficient large values of $m$ and $n$, general values of $p^{0}(d)$ and $\Delta$, a much lower complexity is obtained by using the proposed reverse I-CA scheme. That is, $\mathcal{O}\left(n^{m}\right)>\mathcal{O}\left(n\left(2^{m}-1\right)+p^{0}(d) / \Delta\right)$. If we constrain the number of D2D pairs sharing the same channel to one, the complexity would be further reduced to $\mathcal{O}\left(n \cdot m+p^{0}(d) / \Delta\right)$. And the performance of this reduced scheme is included in the simulation in Subsection VI-A.

\section{E. Overhead}

In D2D underlay system, the BS is still the control center of resource allocation, and the global CSI should indeed be available at the BS for the proposed scheme. In addition to the CSI detection, feedback, and the control signaling transmission, the reverse I-CA scheme does not need additional signaling overhead compared to existing resource scheduling schemes 
such as maximum carrier to interference (Max C/I) and proportional fair (PF), which also need the global CSI to optimize the system performance. The difference is that the reverse ICA scheme requires more complicated CSI due to the interference between D2D and cellular network.

At the beginning of the allocation, the transmitters need to send some packets containing detection signals. Then, the obtained CSI at each terminal (D2D or cellular receiver) would be feedback to the BS. After that, iteration process would be conducted at the BS, and no signaling needs to be exchanged among the network nodes until the control signals forwarding.

Methods, such as CSI feedback compression and signal flooding, would help reduce the overhead. In addition, the future work on D2D communication could consider some mechanism that limit the number of D2D pairs sharing the same channel by, e.g. distance constraint, which would obviously help reduce the overhead. But for this paper, the target is to obtain the nearest-optimal solution, wherefore we do not consider the simplification.

\section{Simulation Results And Discussions}

In this section, we provide the simulation results to illustrate the performances of the proposed reverse I-CA algorithm. Besides, we give the necessary analysis for the results. The main simulation parameters are listed in Table II As shown in Fig. 11 simulations are carried out in a single cell. Both path-loss model and shadow fading are considered for cellular and D2D links. The wireless propagation is modeled according to WINNER II channel models [27], and D2D channel is based on office/indoor scenario while cellular channel is based on the urban microcell scenario.

\section{A. System Sum Rate}

The system sum rate with different numbers of D2D pairs and different numbers of resource units using the proposed auction algorithm is illustrated in Fig. $2 \sim$ Fig. 4, The sum rate can be obtained from (9).

From Fig. 2 and Fig. 4, we can see that the system sum rate goes up with both the number of D2D pairs and the number of resource units increasing. On one side, when the amount 
of resources is fixed, more D2D users contribute to a higher system sum rate. On the other side, as the amount of resource increases, the probability of resources with less interference to D2D links being assigned to them enhances, which can lead to the increased sum rate. This phenomenon is similar to the effect of multiuser diversity. Definitely, cellular users also contributes to the performance.

From another perspective, Fig 2$]$ Fig. 4 shows the system sum rate for different allocation algorithms. The curve marked exhaustive optimal is simulated by the exhaustive search way, which guarantees a top bound of the system sum rate. The curve marked reduced R-I-CA is the result of a reduced reverse I-CA scheme, in which the number of D2D pairs sharing the same cellular resources is constrained to one. The curve marked R-I-CA represents the performance of the proposed reverse I-CA algorithm, and the last one is the simulation result using random allocation of spectrum resources. Firstly, we can see that the proposed auction algorithm is relatively much superior to the random allocation. Secondly, the optimal allocation results in the highest system sum rate, but the superiority compared to R-I-CA is quite small, especially when the number of cellular resource units increases as Fig. 4 shows. Moreover, we find that the performance of reduced R-I-CA approximates to that of R-I-CA scheme in case of 8 resource units, but differs obviously in case of 2 resource units shown in Fig. 3. The reason for this phenomenon is that the constraint of the reduced R-I-CA limits D2D pairs accessing to the network when the number of resources units is less than that of D2D pairs, thus a large capacity loss products.

\section{B. System Efficiency}

We define the system efficiency as $\eta=\Re / \Re_{o p t}$, where $\Re_{o p t}$ represents the exhaustive optimal sum rate. Fig. 5 shows the system efficiency with different numbers of D2D pairs and different numbers of resource units. The simulation result indicates that the proposed algorithm provides high (the lowest value of $\eta$ is around 0.7) system efficiency. Moreover, the efficiency is stable over different parameters of users and resources.

As to the point of efficiency value being about 0.7 , the number of resource units and the number of D2D pairs are both small. The linear price rule limits bidders to bid the maximal 
valuation packages, but to bid the packages having maximal average unit valuation. For this reason, the efficiency decreases slightly.

As to other points, the efficiency is stable above 0.9 , which reflects a small performance gap between the proposed algorithm and the exhaustive search scheme. In fact, the descending price rule determines the bidder that has the highest bid on current items would win the corresponding package, which maximizes the current overall gain. However, the gap cannot be avoided as the algorithm essentially follows a local, or an approximate global optimum principle.

\section{Price Monotonicity}

Fig. 6 shows an example of the price non-monotonicity in the reverse I-CA scheme. The four curves represent unit price of four D2D pairs. As the enlarged detail shows, the unit price of D2D pair 2 has an ascending process during the auction. As the step $\delta$ is much less than descending step $\Delta$, the phenomenon of ascending price is hard to pick out. When the items have been sold out, their prices are fixed to the selling value. And from the figure, we can find that the D2D pair 2 is the last one to be sold.

\section{CONCLUSIONS}

In this paper, we have investigated how to reduce the effects of interference between D2D and cellular users, in order to improve the system sum rate for a D2D underlay network. We have proposed the reverse iterative combinatorial auction as the mechanism to allocate the spectrum resources for D2D communications with multiple user pairs. We have formulated the valuation of each D2D pair for each resource unit, and then explained a detailed auction algorithm depending on the utility function. A non-monotonic descending price iteration process has been modeled and analyzed to be cheat-proof, converge in a finite number of rounds, and has low complexity. The simulation results show that the system sum rate goes up with both the number of D2D pairs and the number of resource units increasing. The proposed auction algorithm is much superior to the random allocation, and provides high system efficiency, which is stable over different parameters of users and resources. 


\section{REFERENCES}

[1] K. Doppler, M. Rinne, C. Wijting, C. Ribeiro, and K. Hugl, "Device-to-device communication as an underlay to LTE-advanced networks," IEEE Commun. Mag., vol. 47, no. 12, pp. 42-49, Dec. 2009.

[2] S. Basagni, M. Conti, S. Giordano, and I. Stojmenovic, Mobile Ad Hoc Networking, pp. 69-116, Wiley-IEEE Press, 2004.

[3] C.-H. Yu, O. Tirkkonen, K. Doppler, and C. Ribeiro, "On the performance of device-to-device underlay communication with simple power control," in Proc. IEEE Vehicular Technology Conference 2009-Spring, Barcelona, Apr. 2009.

[4] J. Zhang, and G. de la Roche, Femtocells: Technologies and Deployment, John Wiley \& Sons Ltd., Nov. 2009.

[5] T. Koskela, S. Hakola, T. Chen, and J. Lehtomaki, "Clustering concept using device-to-device communication in cellular system," in Proc. IEEE Wireless Communications and Networking Conference, Sydney, Apr. 2010.

[6] K. Doppler, M. Rinne, P. Janis, C. Ribeiro, and K. Hugl, "Device-to-device communications; functional prospects for LTE-advanced networks," IEEE International Conference on Communications Workshops, Dresden, Jun. 2009.

[7] K. Doppler, C.-H. Yu, C. Ribeiro, and P. Janis, "Mode selection for device-to-device communication underlaying an LTE-advanced network," IEEE Wireless Communications and Networking Conference, Sydney, Apr. 2010.

[8] H. Min, W. Seo, J. Lee, S. Park, and D. Hong, "Reliability improvement using receive mode selection in the deviceto-device uplink period underlaying cellular networks," IEEE Transactions on Wireless Communications, vol. 10, no. 2, pp. 413-418, Feb. 2011.

[9] S. Hakola, C. Tao, J. Lehtomaki, and T. Koskela, "Device-to-device (D2D) communication in cellular network performance analysis of optimum and practical communication mode selection," IEEE Wireless Communications and Networking Conference, Sydney, Apr. 2010.

[10] C.-H. Yu, K. Doppler, C. Ribeiro, and O. Tirkkonen, "Performance impact of fading interference to Device-to-Device communication underlaying cellular networks," IEEE 20th International Symposium on Personal, Indoor and Mobile Radio Communications, pp. 858-862, Tokyo, Sept. 2009.

[11] C.-H. Yu, O. Tirkkonen, K. Doppler, and C. Ribeiro, "Power optimization of device-to-device communication underlaying cellular communication," in Proc. IEEE International Conferemce on Communications, Dresden, Jun. 2009.

[12] H. Xing, and S. Hakola, "The investigation of power control schemes for a device-to-device communication integrated into OFDMA cellular system," IEEE 21st International Symposium on Personal Indoor and Mobile Radio Communications, pp. 1775-1780, Instanbul, Sept. 2010.

[13] P. Janis, V. Koivunen, C.B. Ribeiro, K. Doppler, and K. Hugl, "Interference-avoiding MIMO schemes for deviceto-device radio underlaying cellular networks," IEEE 20th International Symposium on Personal, Indoor and Mobile Radio Communications, pp. 2385-2389, Tokyo, Sept. 2009.

[14] T. Peng, Q. Lu, H. Wang, S. Xu, and W. Wang, "Interference avoidance mechanisms in the hybrid cellular and deviceto-device systems," IEEE 20th International Symposium on Personal, Indoor and Mobile Radio Communications, pp. 617-621, Tokyo, Sept. 2009.

[15] P. Janis, V. Koivunen, C. Ribeiro, J. Korhonen, K. Doppler, and K. Hugl, "Interference-aware resource allocation for device-to-device radio underlaying cellular networks," in Proc. IEEE Vehicular Technology Conference 2009-Spring, Barcelona, Apr. 2009.

[16] M. Zulhasnine, C. Huang, and A. Srinivasan, "Efficient resource allocation for device-to-device communication underlaying LTE network," in IEEE 6th International Conference on Wireless and Mobile Computing, Networking and Communications, pp. 368-375, Niagara Falls, Oct. 2010.

[17] S. Xu, H. Wang, T. Chen, Q. Huang, and T. Peng, "Effective interference cancellation scheme for device-to-device 
communication underlaying cellular networks," in Proc. IEEE Vehicular Technology Conference 2010-Fall, Ottawa, Sept. 2010.

[18] C.-H. Yu, K. Doppler, C. Ribeiro, and O. Tirkkonen, "Resource sharing optimization for D2D communication underlaying cellular networks,” IEEE Trans. Wireless Commmun., vol. 10, no. 8, pp. 2752-2763, Aug. 2011.

[19] D. Fudenberg and J. Tirole, Game Theory, MIT Press, Cambridge, MA, 1993.

[20] J. Bae, E. Beigman, R. Berry, M. L. Honig, and R. Vohra, "Sequential bandwidth and power auctions for distributed spectrum sharing," IEEE Journals on Selected Areas in Communications, vol. 26, no. 9, pp. 1193-1203, Sept. 2008.

[21] A. Das, and D. Grosu, "Combinatorial auction-based protocols for resource allocation in grids," in Proc. IEEE International Parallel and Distributed Processing Symposium, Apr. 2005.

[22] H. C. Lau, S. F. Cheng, T. Y. Leong, J. H. Park, and Z. Zhao, "Multi-period combinatorial auction mechanism for distributed resource allocation and scheduling," IEEE/WIC/ACM International Conference on Intelligent Agent Technology, pp.407-411, Fremont, Nov. 2007.

[23] P. Cramton, Y. Shoham, and R. Steinberg, Combinatorial Auctions, MIT Press, 2005.

[24] A. Pikovsky, Pricing and Bidding Strategies in Iterative Combinatorial Auctions, Ph.D. Dissertation, Munich, Germany, 2008.

[25] M. Bichler, P. Shabalin, and A. Pikovsky, "A computational analysis of linear price iterative combinatorial auction formats," Information Systems Research, vol. 20, no. 1, pp. 33-59, Mar. 2009.

[26] C. Xu, L. Song, Z. Han, Q. Zhao, X. Wang, and B. Jiao, "Interference-Aware Resource Allocation for Device-toDevice Communications as an Underlay Using Sequential Second Price Auction," IEEE Internetional Conference on Communications, Ottawa, Jun. 2012.

[27] WINNER II D1.1.2, “WINNER II channel models,” https://www.istwinner.org/deliverables.html, Sept. 2007.

[28] ITU-R M.2135-1, "Guidelines for evaluation of radio interface technologies for IMT-Advanced," http://www.itu.int/pub/R-REP-M.2135-1-2009, Dec. 2009.

[29] M. Haenggi, J. G. Andrews, F. Baccelli, O. Dousse, and M. Franceschetti, "Stochastic geometry and random graphs for the analysis and design of wireless networks," IEEE Journal on Selected Areas in Communications, vol. 27, no. 7, pp. 1029-1046, Sept. 2009.

[30] Z. Han, and K. J. Ray Liu, Resource Allocation for Wireless Networks: Basics, Techniques, and Applications, Cambridge University Press, 2008.

[31] "Selection procedures for the choice of radio transmission technologies of the UMTS," 3GPP TR 30.03U, version 3.2.0, 1998. 
TABLE I

THE RESOURCE ALLOCATION ALGORITHM

\section{* Initial State:}

The BS collects the location information of all D2D pairs. The valuation of the $c$-th resource unit for package $k$ is $v_{c}(k), c=1,2, \ldots, C, k=1,2, \ldots, N$, which is given by (14). The round index $t=0$, and the initial price $P^{0}(d)$, the fixed price reduction $\Delta>0$ are set up.

\section{* Resource Allocation Algorithm:}

1. Bidder $c$ submits bids $\left\{\mathcal{D}_{k}, \mathcal{P}_{c}(k)\right\}$ depending on its utility.

$\star$ bidder $c$ bids for package $\mathcal{D}_{k}$ as long as $\mathcal{U}_{c}(k) \geq 0$, which is represented by (24).

$\star$ If $\mathcal{U}_{c}(k)<0$, bidder $c$ submits $\{\emptyset, 0\}$.

2. If $\exists d \in \mathcal{D}_{k}$ satisfies $\forall b_{c}^{t}(k) \in \mathcal{B}^{t}, \mathcal{D}_{k} \notin b_{c}^{t}(k)$, the BS sets $t=t+1, p^{t+1}(d)=p^{t}(d)-\Delta$ where $d$ is the over-supplied item, and the auction moves on to the next round. Return to step 1.

3. The BS detects the bids of all the bidders:

1) it exists $b_{c_{1}}^{t}(k)=b_{c_{2}}^{t}(k) \neq\{\emptyset, 0\}\left(c_{1} \neq c_{2}, k \in\{1,2, \ldots, N\}\right)$;

2) it exists $b_{c_{1}}^{t}\left(k_{1}\right)=\left\{\mathcal{D}_{k_{1}}, \mathcal{P}_{c_{1}}^{t}\left(k_{1}\right)\right\}, b_{c_{2}}^{t}\left(k_{2}\right)=\left\{\mathcal{D}_{k_{2}}, \mathcal{P}_{c_{2}}^{t}\left(k_{2}\right)\right\}\left(k_{1} \neq k_{2}, c_{1}, c_{2} \in\right.$ $\{1,2, \ldots, C\}$ ) satisfying $\mathcal{D}_{k_{1}} \cap \mathcal{D}_{k_{2}} \neq \emptyset$.

4. If neither of the conditions in step 3 is satisfied, go to step 5. Otherwise, the overall demand exceeds supply for at least one item. The BS sets $p^{t}(d)=p^{t}(d)+\delta$, and $\delta$ can be set by $\delta=\Delta / i$ where $i$ is an integer factor. Return to step 1 .

5. The allocation can be determined by repeating the above steps. The auction continues until all D2D links are auctioned off or every cellular channel wins a package.

TABLE II

Main Simulation Parameters

\begin{tabular}{|l|l|}
\hline Parameter & Value \\
\hline Cellular layout & Isolated cell, 1-sector \\
\hline System area & The radius of the cell is $500 \mathrm{~m}$ \\
\hline Noise spectral density & $-174 \mathrm{dBm} / \mathrm{Hz}$ \\
\hline Sub-carrier bandwidth & $15 \mathrm{kHz}$ \\
\hline Noise figure & $9 \mathrm{~dB}$ at device \\
\hline Antenna gains & BS: $14 \mathrm{dBi}$; Device: $0 \mathrm{dBi}$ \\
\hline The maximum distance of D2D & $5 \mathrm{~m}$ \\
\hline Transmit power & BS: $46 \mathrm{dBm}$; Device: $23 \mathrm{dBm}$ \\
\hline
\end{tabular}




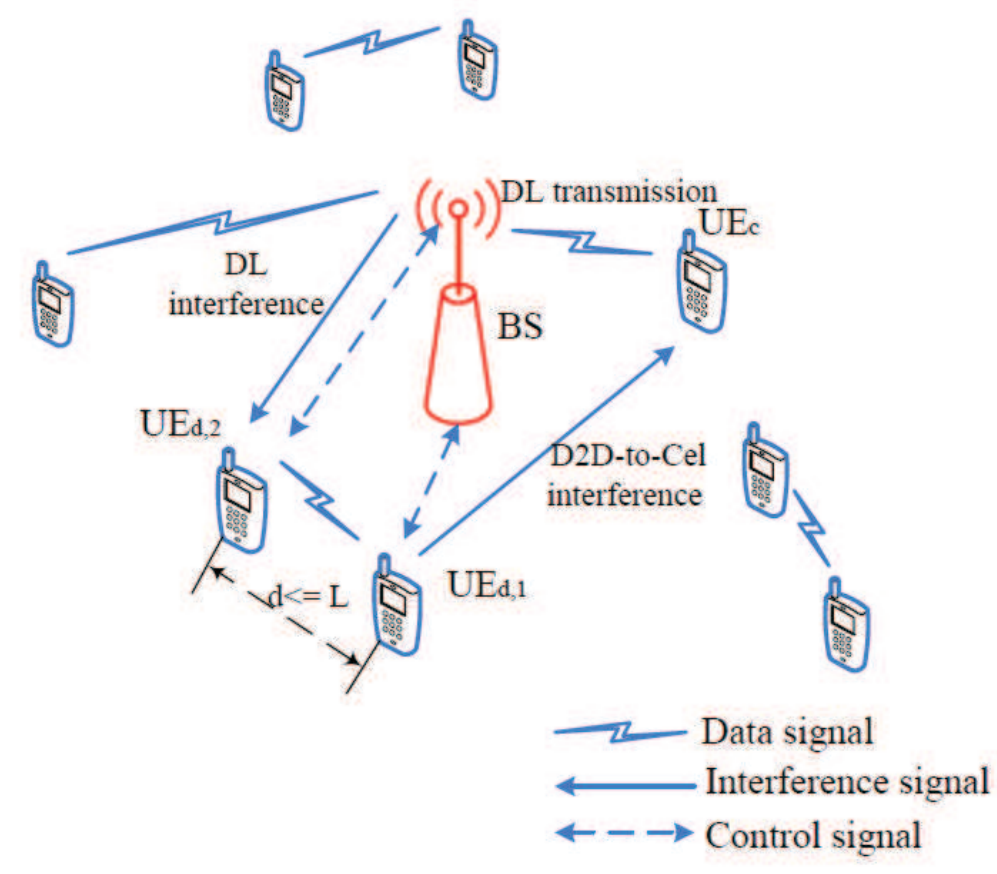

Fig. 1. System model of D2D communication underlaying cellular networks with downlink resource sharing.

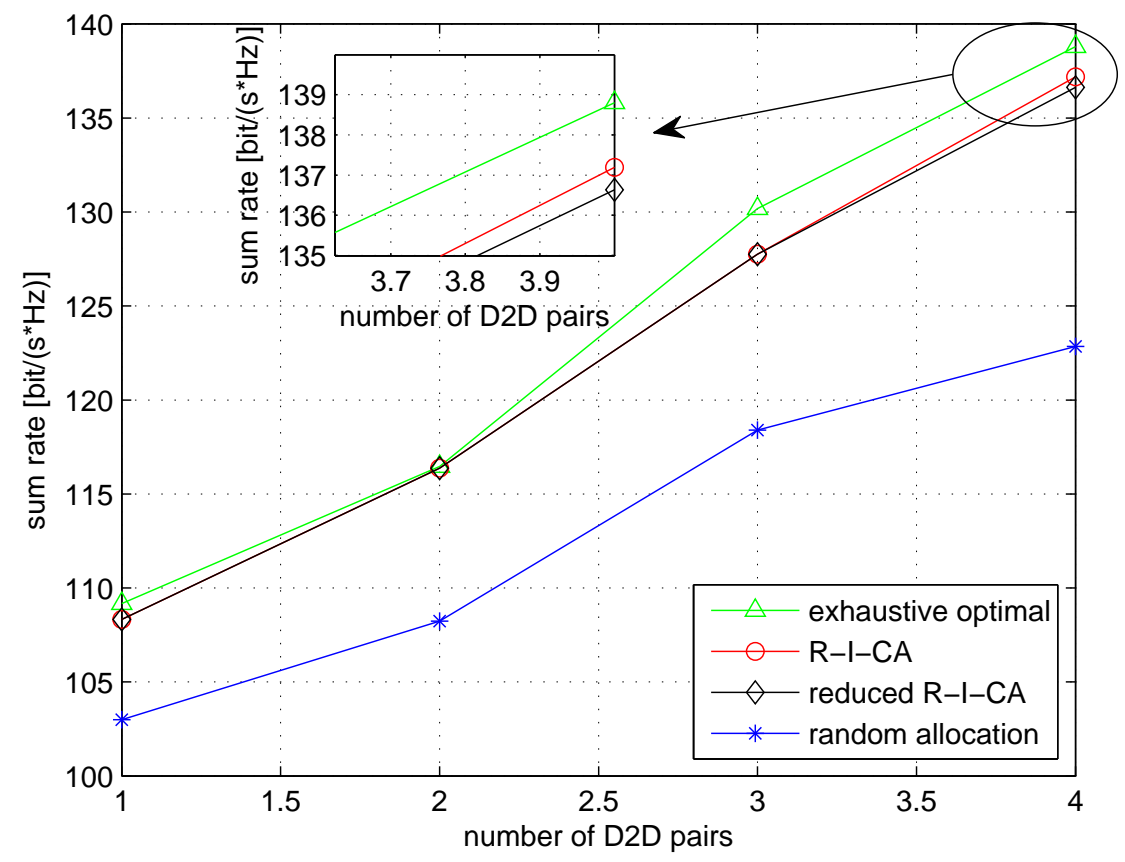

Fig. 2. System sum rate for different allocation algorithms in the case of 8 resource units. 


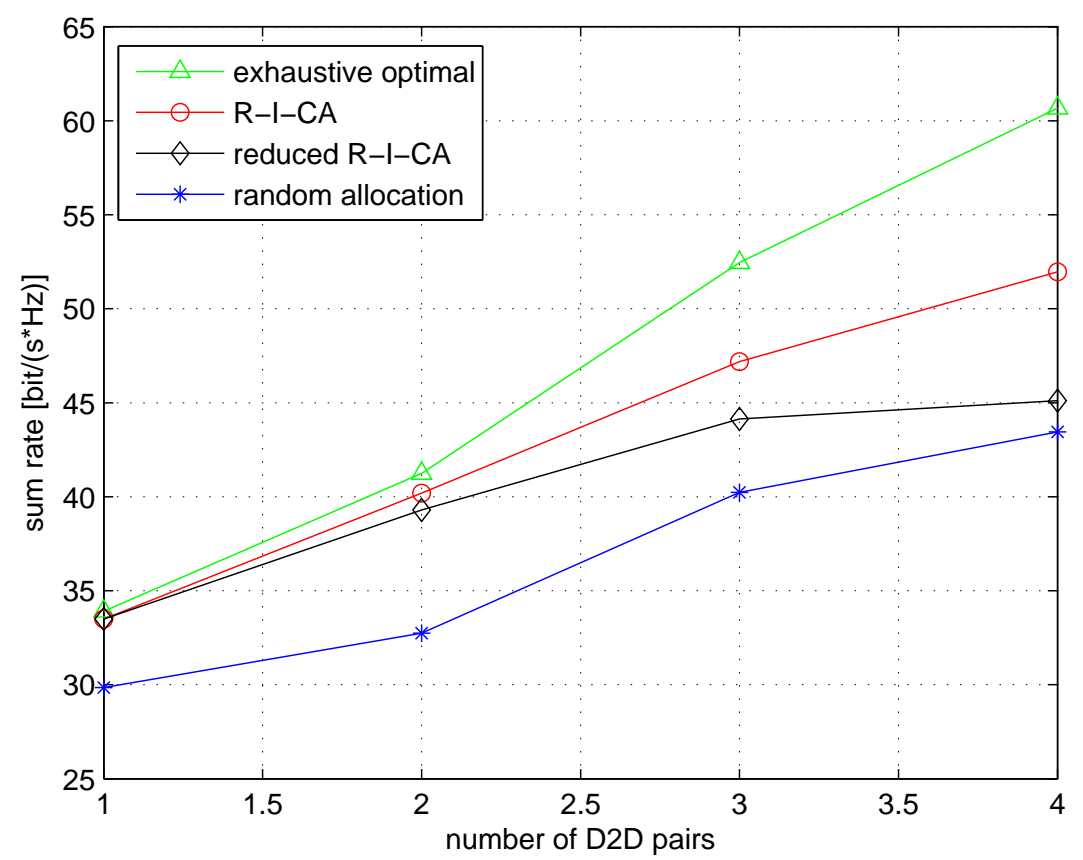

Fig. 3. System sum rate for different allocation algorithms in the case of 2 resource units.

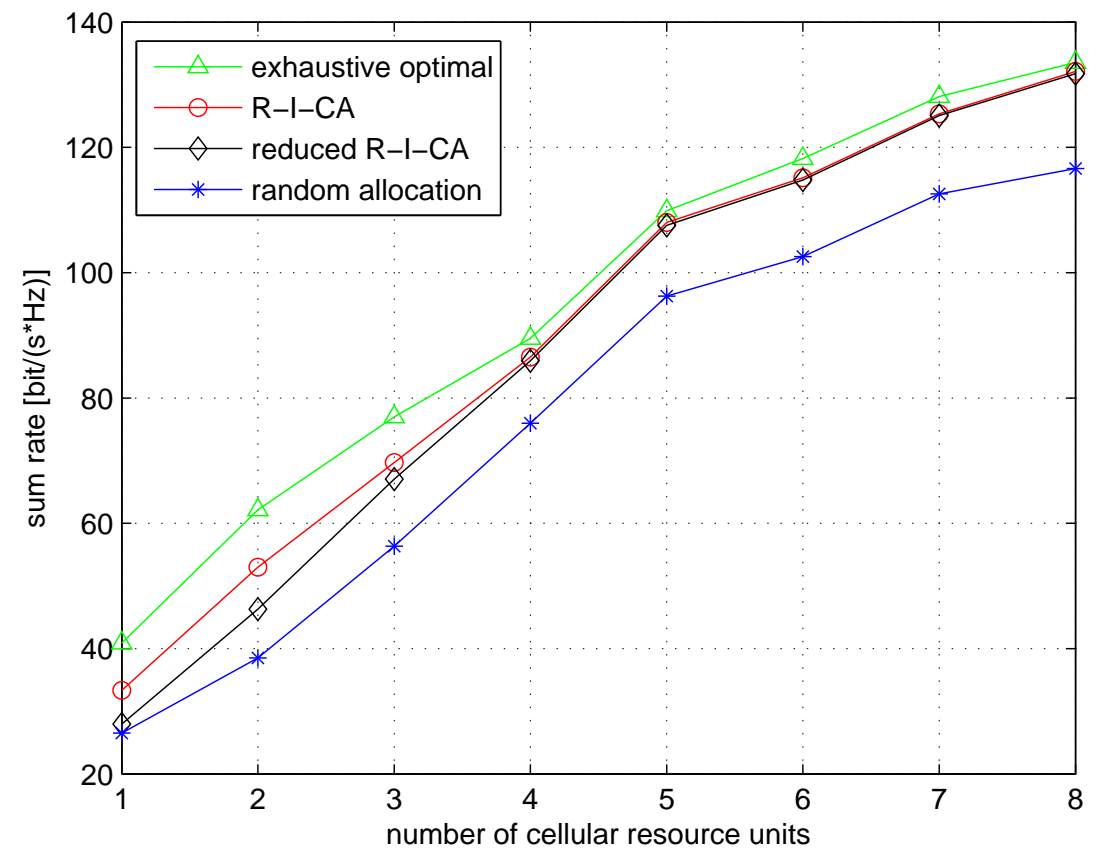

Fig. 4. System sum rate for different allocation algorithms in the case of 4 D2D pairs. 


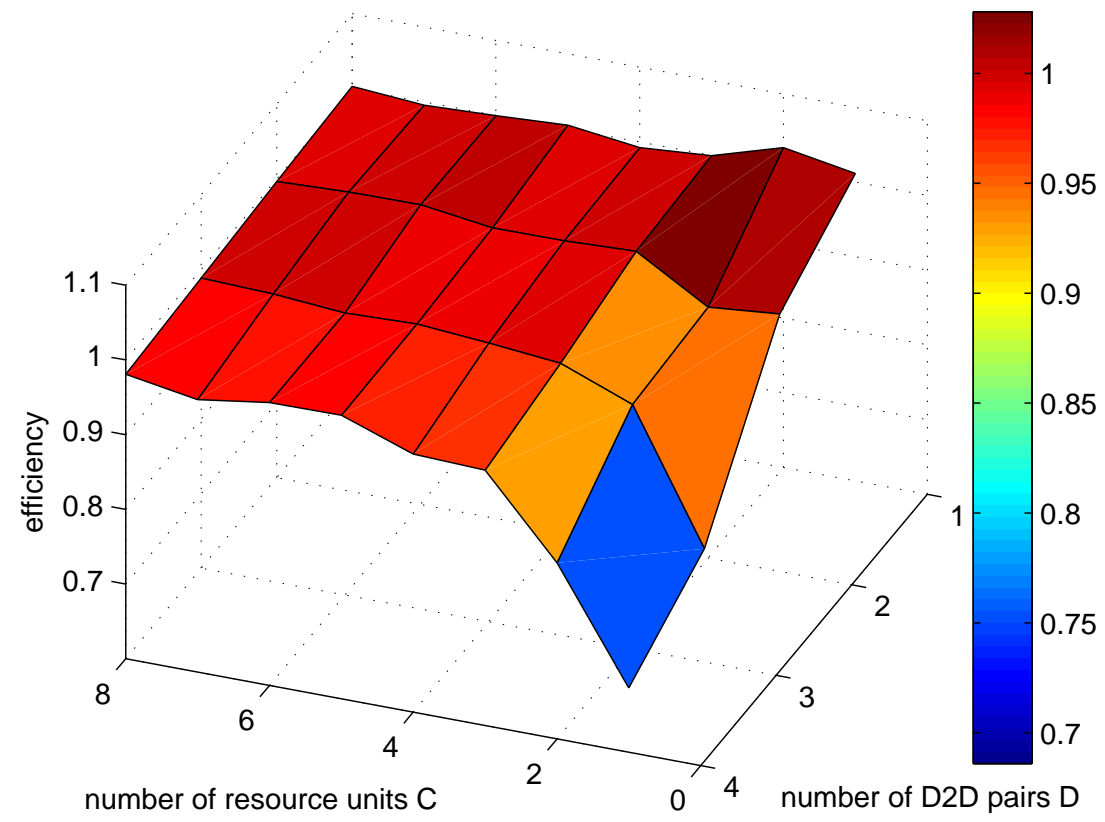

Fig. 5. System efficiency: $\eta$ with different numbers of D2D pairs and different numbers of resource units.

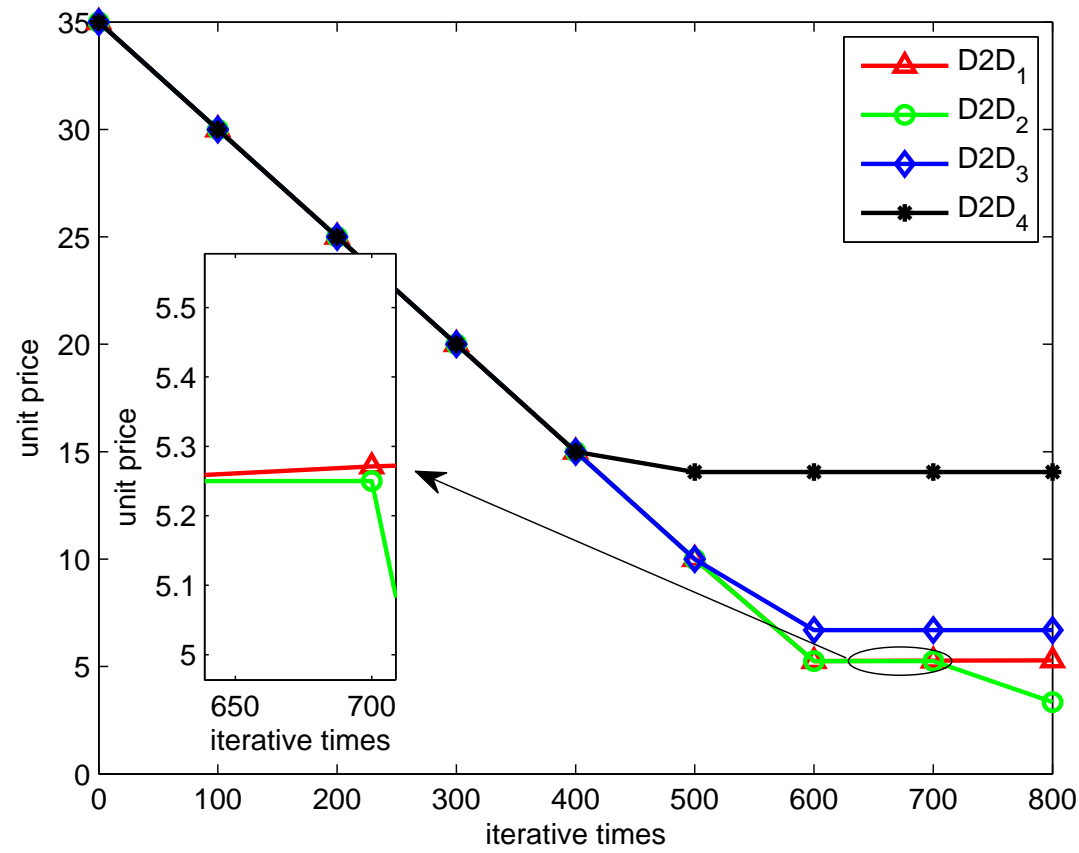

Fig. 6. Price monotonicity: an example of price non-monotonicity in the reverse I-CA scheme. 